\title{
Dark photon search at Yemilab, Korea
}

\author{
S.H. Seo, ${ }^{a, 1,2}$ and Y.D. Kim ${ }^{a, b, c, 3}$ \\ ${ }^{a}$ Center for Underground Physics, Institute for Basic Science (IBS), \\ 55, Expo-ro, Yuseong-gu, Daejeon, 34126, Korea \\ ${ }^{b}$ Department of Physics and Astronomy, Sejong University, \\ Seoul, 05006, Korea \\ ${ }^{c}$ IBS School, University of Science and Technology (UST), \\ Daejeon, 34113, Korea \\ E-mail: sunny.seo@ibs.re.kr, ydkim@ibs.re.kr
}

ABSTRACT: Dark photons are well motivated hypothetical dark sector particles that could account for observations that cannot be explained by the standard model of particle physics. A search for dark photons that are produced by an electron beam striking a thick tungsten target and subsequently interact in a 3 kiloton-scale neutrino detector in Yemilab, a new underground lab in Korea, is proposed. Dark photons can be produced by "darkstrahlung" or by oscillations from ordinary photons produced in the target and detected by their visible decays, "absorption" or by their oscillation to ordinary photons. By detecting the absorption process or the oscillation-produced photons, a world's best sensitivity for measurements of the dark-photon kinetic mixing parameter of $\epsilon^{2}>1.5 \times 10^{-13}\left(6.1 \times 10^{-13}\right)$ at the $95 \%$ confidence level (C.L.) could be obtained for dark photon masses between $80 \mathrm{eV}$ and $1 \mathrm{MeV}$ in a year-long exposure to a $100 \mathrm{MeV}-100 \mathrm{~kW}$ electron beam with zero $\left(10^{3}\right)$ background events. In parallel, the detection of $e^{+} e^{-}$pairs from decays of dark photons with mass between $1 \mathrm{MeV}$ and $\sim 86 \mathrm{MeV}$ would have sensitivities of $\epsilon^{2}>\mathcal{O}\left(10^{-17}\right)\left(\mathcal{O}\left(10^{-16}\right)\right)$ at the $95 \%$ C.L. with zero $\left(10^{3}\right)$ background events. This is comparable to that of the Super-K experiment under the same zero background assumption.

KeYwords: Beyond Standard Model, Other experiments

ARXIV EPRINT: 2009.11155

\footnotetext{
${ }^{1}$ Corresponding author.

${ }^{2}$ https://orcid.org/0000-0002-1496-624X.

${ }^{3}$ https://orcid.org/0000-0003-2471-8044.
} 


\section{Contents}

1 Introduction 1

2 A neutrino detector for Yemilab 2

3 Dark photon production, detection and expected number 5

3.1 Dark photon production 5

$\begin{array}{lll}3.2 & \text { Dark photon detection } & 6\end{array}$

$\begin{array}{lll}3.3 & \text { Expected number of dark photons } & 7\end{array}$

4 Dark photon sensitivity at Yemilab $\quad 9$

$\begin{array}{lll}\text { 4.1 Dark photon decay and absorption lengths } & 9\end{array}$

$\begin{array}{llr}4.2 & \text { Background estimation } & 9\end{array}$

$\begin{array}{lll}4.3 & \text { Dark photon sensitivity } & 12\end{array}$

$\begin{array}{lll}4.4 & \text { Oscillation between ordinary and dark photons } & 13\end{array}$

$\begin{array}{llr}5 & \text { Summary } & 16\end{array}$

\section{Introduction}

The standard model of particle physics has been very successful at explaining phenomena in the visible universe. However, it has a number of clear limitations. It provides no explanations for dark matter, the muon g- 2 anomaly, the $m_{e e}=17 \mathrm{MeV} e^{+} e^{-}$excess from ${ }^{8} \mathrm{Be}$ that is reported in refs. $[1,2]$, etc. In order to explain these "beyond the standard model" (BSM) hints of new physics, the introduction of a hypothetical sector of new particles and interactions, the dark (or hidden) sector has been proposed. In this scheme, there are only a few portals (or mediators) that connect the dark sector to the visible universe that have significant strength and satisfy Lorentz and gauge symmetries. These are the vector, higgs, neutrino, and axion portals that can be explored with different types of experiments.

In particular, in the vector portal, a dark photon (usually denoted as $A^{\prime}, \phi$, or $\gamma^{\prime}$ ), that usually described by an extra $\mathrm{U}(1)_{D}$ gauge symmetry group, is the dark sector particle that could be more readily explored than corresponding particles in the other portals because it can kinetically mix with an ordinary photon. As a result, any experiment that can produce photons and detect photons or leptons can, in principle, explore the vector portal [3].

The Lagrangian that describes the dark photon (DP) is

$$
\mathcal{L} \supset-\frac{1}{4} F_{\mu \nu}^{\prime} F^{\prime \mu \nu}+\frac{\epsilon}{2} F_{\mu \nu}^{\prime} F^{\mu \nu}+\frac{m_{\phi}^{2}}{2} A_{\mu}^{\prime} A^{\prime \mu},
$$

where $F_{\mu \nu}^{\prime} \equiv \partial_{\mu} A_{\nu}^{\prime}-\partial_{\nu} A_{\mu}^{\prime}$ is the DP field strength tensor, $A_{\mu}^{\prime}$ is the $\mathrm{U}(1)_{D}$ gauge field, $\epsilon$ is the kinetic-mixing strength between the dark and ordinary photons, and $m_{\phi}$ is the dark photon mass. 


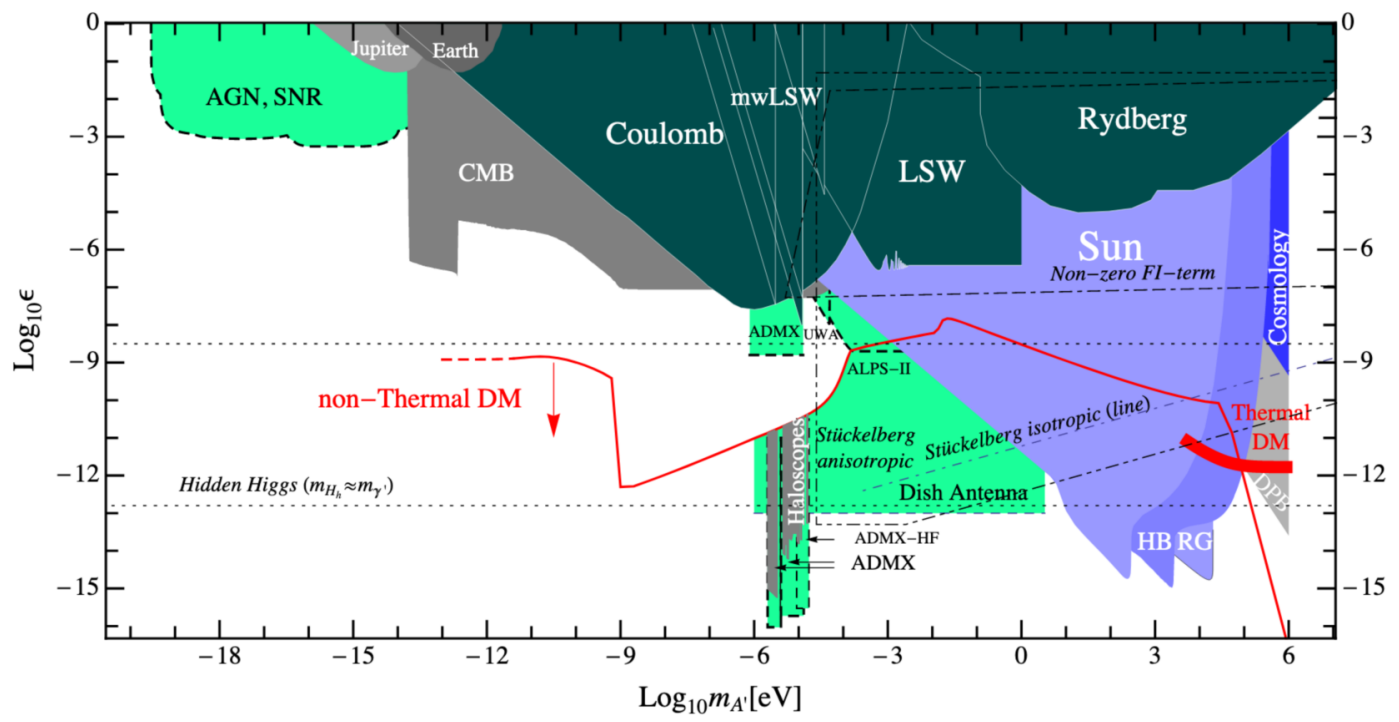

Figure 1. Current limits on $\epsilon$ for $m_{\phi}<2 m_{e}$ dark photons from laboratory search experiments and astrophysical observations (from ref. [32]).

The 1988 SLAC beam dump experiment (E137) was the pioneering search for dark photons [4]. More recently, in the last decade, there have been numerous reports of dark photon searches [5-29] based on data from fixed target accelerator experiments, $e^{+} e^{-}$ colliders, reactors and astrophysical measurements, that have set stringent limits to the dark photon parameter space. These limits could be further improved or, possibly, a darkphoton signal could be discovered, by current or future experiments without huge costs. Figures 1 and 2 show the current constraints (or sensitivities) on $\epsilon$ for $m_{\phi}<2 m_{e}$ and $m_{\phi}>2 m_{e}$, respectively; comprehensive reviews on the status of dark photon searches can be found in refs. [30, 31].

Yemilab, a new underground lab that is being constructed in Handuk iron mine in Jeongseon-gun, Korea, will have a cavern that will be capable of hosting a $\sim 3$ kiloton liquid target neutrino detector. A $100 \mathrm{MeV}$ electron accelerator $(100 \mathrm{~kW}$ or $10 \mathrm{~kW}$ beam power) located close to the neutrino detector, would make a dark photon search possible at Yemilab.

In the following sections, the proposed Yemilab neutrino detector 2, expected numbers of produced and detected dark photons 3, and the expected dark photon sensitivity 4 are described. These are followed by a summary 5 .

\section{A neutrino detector for Yemilab}

By early 2022, the $2^{\text {nd }}$ phase construction of Yemilab ( $\sim 1000 \mathrm{~m}$ overburden under the eponymous Mt. Yemi) will be completed and experimental operation will commence (see figure 3). In addition to spaces for the upgraded COSINE [34] dark matter and AMoREII [35] $0 \nu \beta \beta$ search experiments, a cavern suitable for hosting a $\sim 3$ kiloton neutrino detector 

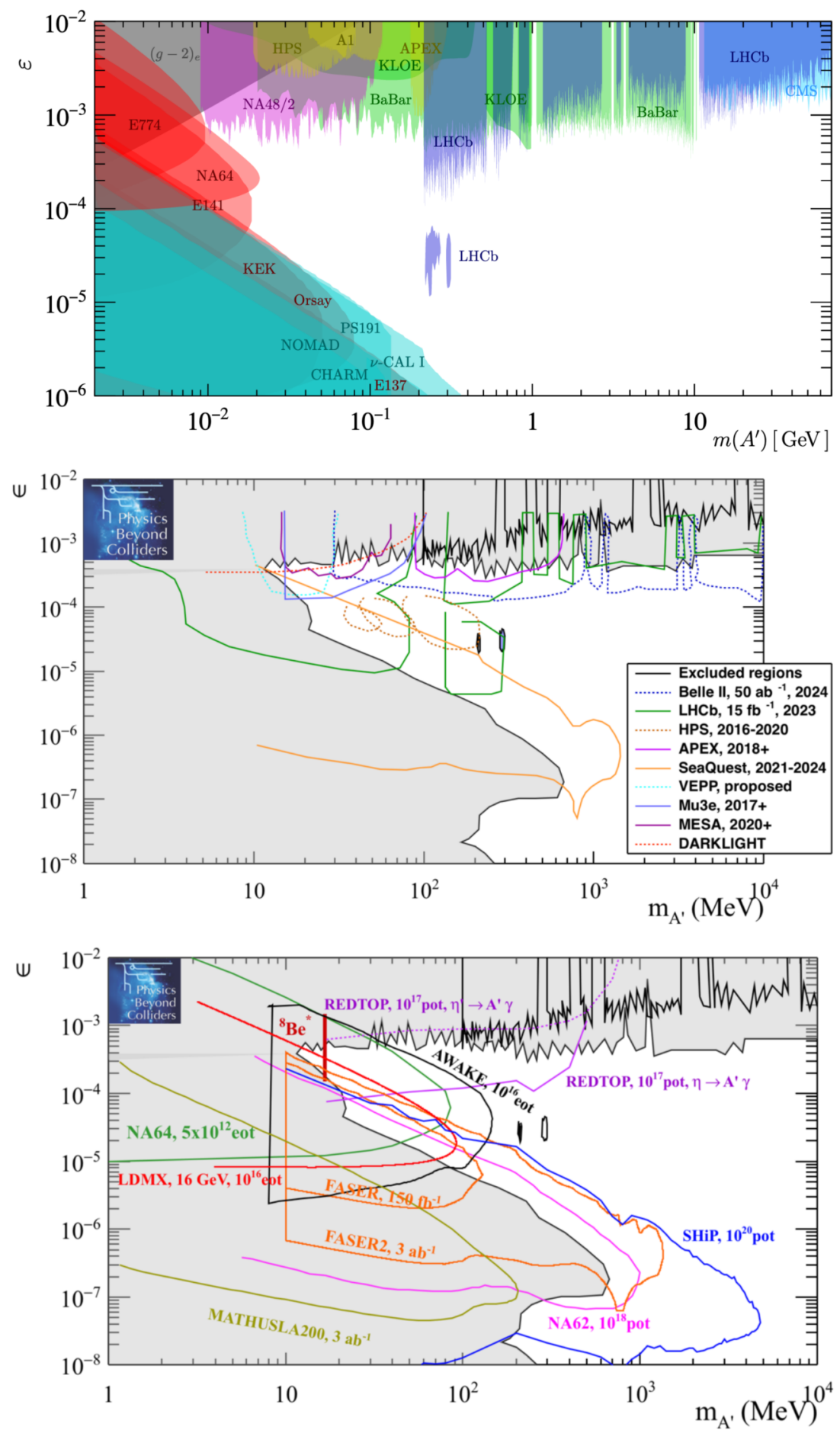

Figure 2. Current limits (color-filled or shaded regions) and sensitivities (solid lines) on $\epsilon$ for $m_{\phi}>2 m_{e}$ dark photons from various experiments. Current limits only (top) from ref. [33], the sensitivities that are expected to be by the experiments/proposals outside Physics Beyond Colliders (PBC) activities (middle), and those within PBC activities (bottom) from ref. [30]. Up-to-date limits can be also directly obtained in a software framework called darkcast [24] at https://gitlab.com/philten/darkcast. 

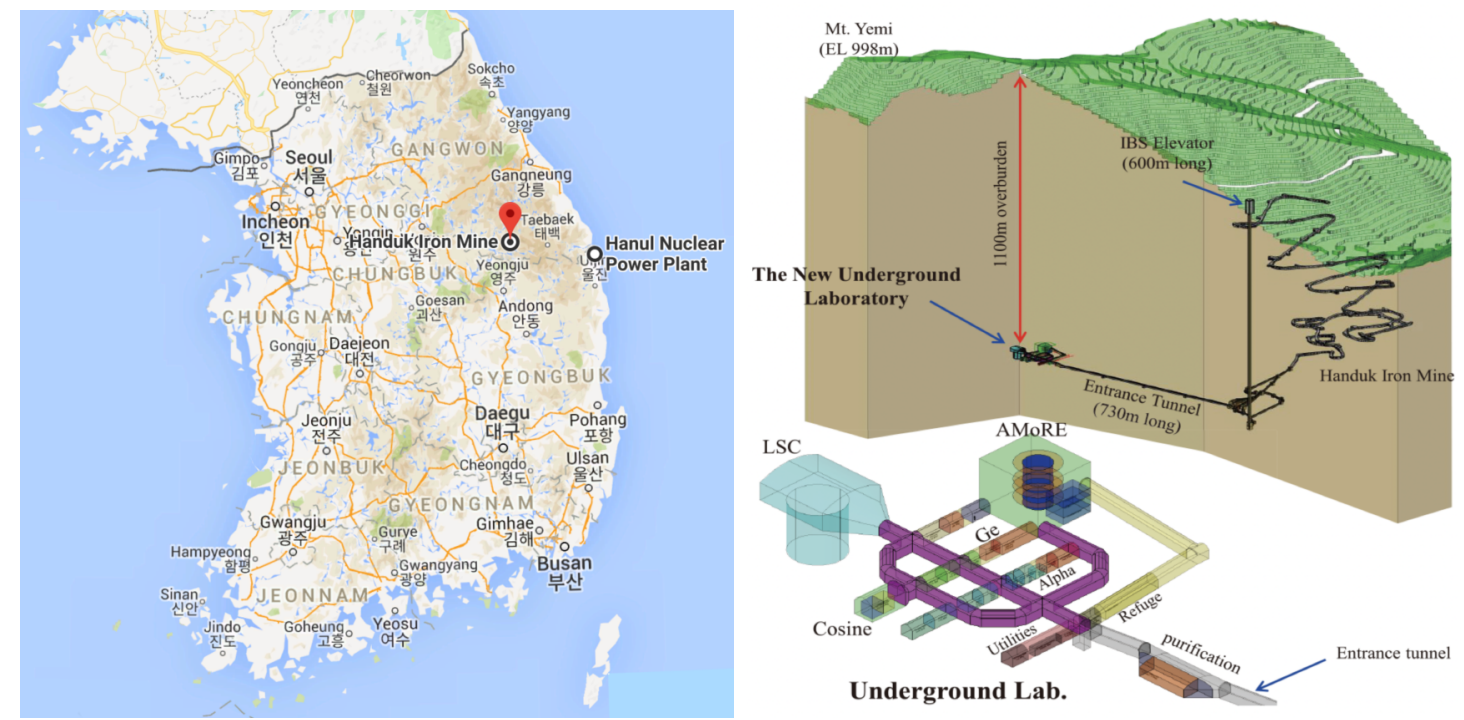

Figure 3. (Left) The Handuk iron mine location (latitude: $37.188639 \mathrm{deg}$, longitude: 128.659406 $\mathrm{deg})$ in Jeongseon-gun, Gangwon province, Korea, where a new underground Yemilab $(\sim 1000 \mathrm{~m}$ overburden) is currently being constructed. (Right) The layout of Yemilab, including a cavern for a $\sim 3$ kiloton neutrino detector (LSC). The laboratory will be accessed by a $600 \mathrm{~m}$ vertical shaft and a $730 \mathrm{~m}$ entrance tunnel. Adapted from ref. [36].

will be available. The current plans for this space include a liquid scintillator (LS) or waterbased LS (WbLS) neutrino detector [36].

The $\sim 3$ kiloton Yemilab neutrino detector is primarily dedicated to precise determinations of solar neutrino fluxes and measurements of geo-neutrinos [36] as well as a sterile neutrino search using a strong radioactive source in a later stage. It would be the first kiloton-scale neutrino telescope in Korea and a follow-on to the successful programs of the smaller scale RENO [37] and NEOS [38] reactor neutrino experiments at the Hanbit Nuclear Power Plant. The Yemilab neutrino detector could also be used to for a dark photon search by the addition of an electron accelerator for underground experiments, as suggested in ref. [39]. Figure 4 is a schematic diagram that shows how an electron linac $(100 \mathrm{MeV}$, $100 \mathrm{~kW}$ or $10 \mathrm{~kW})$, tungsten target \& radiation shield $(50 \mathrm{~cm}$-thick), and the neutrino detector (D: $20 \mathrm{~m}, \mathrm{H}: 20 \mathrm{~m}$ cylinder) could be configured at Yemilab. To prevent dark photon decay particles $\left(e^{+} e^{-}\right.$or $\left.3 \gamma\right)$ occurred outside detector target from being absorbed by the outer shell of the detector or outer detector (i.e., water Cherenkov detector for muon veto and buffer region where PMTs are mounted), a guiding tube penetrating the outer detector up to the target would be installed.

Final state of dark photon signal would be $e^{+} e^{-}$or single (triple) gamma(s) and this is described in the following section. Regardless of the choice of the neutrino target medium between LS and WbLS, our search strategy is inclusive for all possible final states for a visible mode considered in this study, and signal candidates are identified by counting number of PMTs fired or measuring visible energy within pulsed beam time window. Background events are subtracted by beam-ON minus beam-OFF data. 

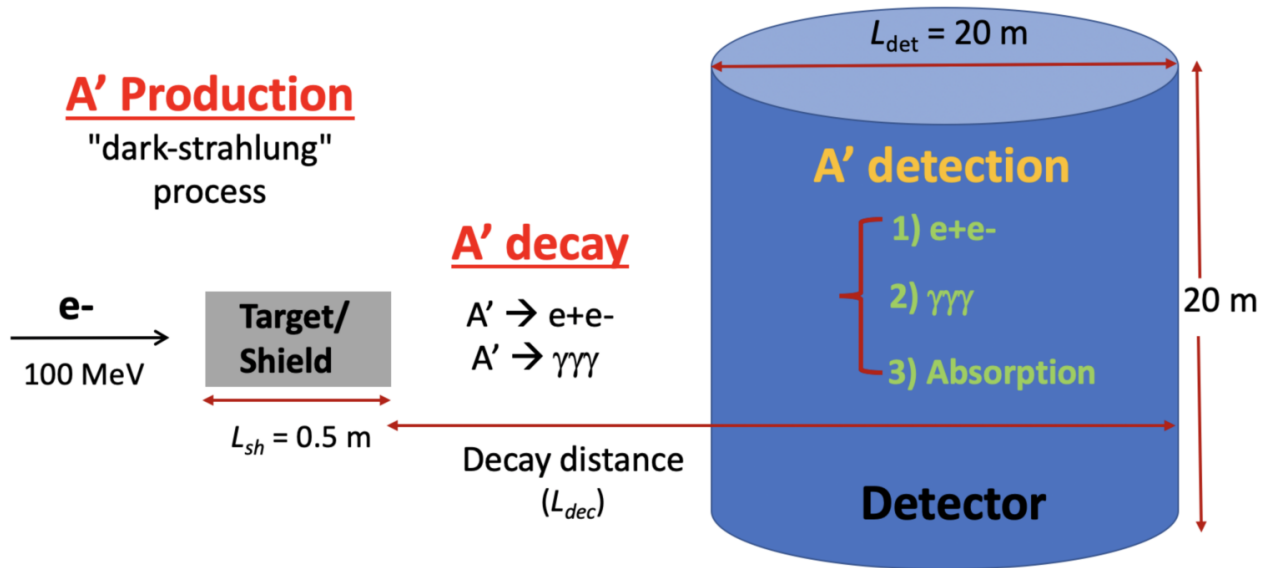

Figure 4. A schematic diagram showing a possible experimental configuration for a dark photon search at Yemilab. The $L_{\mathrm{sh}}\left(L_{\mathrm{det}}\right)$ represents horizontal length of target \& shield (detector) in the direction of $e^{-}$beam. Decay distance, $L_{\mathrm{dec}}$, is set as $20 \mathrm{~m}$ in our study but it is flexible to change.

\section{Dark photon production, detection and expected number}

\subsection{Dark photon production}

In dark sector models with a vector portal there is a small mixing between dark photons and ordinary photons. As a result, dark photons could be produced via the electron bremsstrahlung process that is common for ordinary photons; i.e., when an electron beam strikes a target with atomic number $Z$, dark photons, $A^{\prime}$, are produced by the reaction $e^{-}+Z \rightarrow e^{-}+Z+A^{\prime}$, as illustrated in figure 5. Practical calculations generally use the Weizäscker-Williams (WW) approximation to obtain the dark photon production cross-section via this reaction, in this case known as the "darkstrahlung" process, as a simplification of the exact result, which is quite complicated. For computer calculations an improved WW (IWW) approximation that increases the calculation speed has been also developed and widely used as well. These approximations, however, assume that the dark photon's mass is much greater than electron mass and much less than electron beam energy. Recently, Liu and Miller [40] have reported an exact calculation of the darkstrahlung cross-section and, in addition, generalized versions of the WW and IWW approximations so that no restrictions on the dark photon mass apply. Liu and Miller compared their exact calculation with the generalized IWW approximation that was used by SLAC experiment E137 and found reasonable agreement for dark photon masses below $100 \mathrm{MeV}$.

In this work, the generalized IWW approximation by Liu and Miller is employed to compute darkstrahlung cross-sections. This has the following analytic form

$$
\frac{d \sigma}{d x}=2 \epsilon^{2} \alpha^{3} \chi \frac{|k|}{E} \frac{m_{e}^{2} x\left(-2+2 x+x^{2}\right)-2\left(3-3 x+x^{2}\right) x \tilde{u}_{\max }}{3 x \tilde{u}_{\max }},
$$

where $x$ is the fraction of energy a dark photon carries away from electron energy $(E),|k|$ is the 3 -momentum of the dark photon, $\epsilon$ is the kinetic mixing parameter, $\alpha(\simeq 1 / 137)$ is the fine structure constant, $\tilde{u}_{\max }=-m_{\phi}^{2} \frac{1-x}{x}-m_{e}^{2} x$, and $\chi$ is the effective photon flux and 

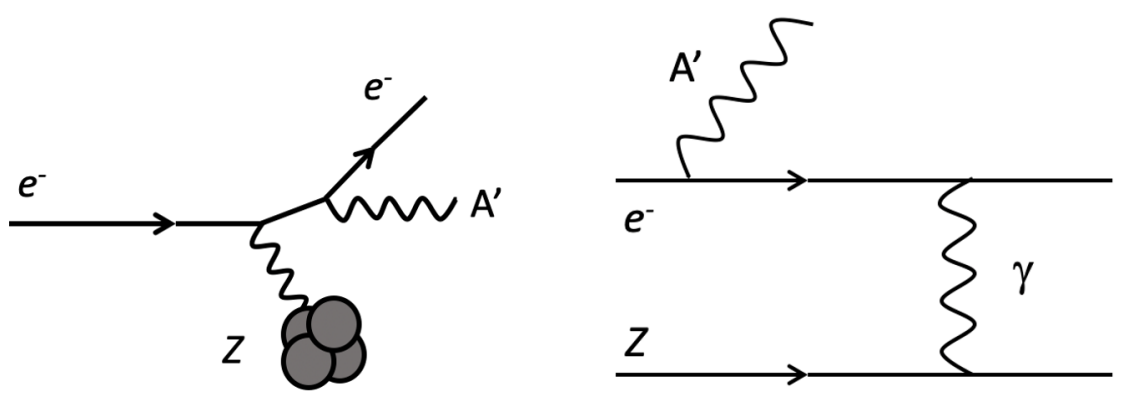

Figure 5. Dark Photon production via the "darkstrahlung" process.

given by

$$
\chi=\int_{t_{\min }}^{t_{\max }} d t \frac{t-t_{\min }}{t^{2}}\left[G_{2, e l}(t)+G_{2, i n}(t)\right]
$$

Here $t$ is the square of the four-momentum transferred to the target nucleus, which ranges from $t_{\min }=\left(\frac{m_{\phi}^{2}}{2 E}\right)^{2}$ to $t_{\max }=m_{\phi}^{2}+m_{e}^{2}$ in the IWW approximation where the produced dark photon is assumed to be collinear to the incident electron, and $G_{2, e l}(t)$ and $G_{2, i n}(t)$ are elastic and inelastic form factors of the target nucleus, respectively. In the following, we only consider the elastic form factor because the contribution from the inelastic one is negligibly small. The $\chi / Z^{2}$ values are shown in figure 10 of ref. [3] for $200 \mathrm{MeV}, 1 \mathrm{GeV}$, and $6 \mathrm{GeV} e^{-}$beams on a tungsten target. For $m_{\phi}<100 \mathrm{MeV}$ with a $200 \mathrm{MeV} e^{-}$beam, the $\chi / Z^{2}$ values range from $\sim 1$ to $\sim 7$ and we infer from the figure that the range of $\chi / Z^{2}$ values for $\mathrm{MeV}$-scale dark photon masses are similar for the case of a $100 \mathrm{MeV} e^{-}$beam. For simplicity, in this work we use $\chi / Z^{2}=6$ and found that changes in this value over a reasonable range has negligible effects on our results.

Figure 6 shows the differential darkstrahlung cross-section for a $100 \mathrm{MeV} e^{-}$beam on a tungsten target as a function of the fractional dark photon (DP) energy, $x$, where the crosssections of the DP masses of $1 \mathrm{keV}, 10 \mathrm{keV}, 100 \mathrm{keV}, 1 \mathrm{MeV}$, and $10 \mathrm{MeV}$ are compared. As the dark photon mass gets heavier, the cross-section decreases as expected from eq. (3.1), while the relative contribution from high $x$ values increases.

\subsection{Dark photon detection}

If $m_{\phi}>2 m_{e}$, dark photons could decay to the visible $e^{+} e^{-}$final state; if $m_{\phi}<2 m_{e}$ the only visible decay mode is the $\gamma \gamma \gamma$ channel. If the DP mass is greater than $2 m_{\mu}$, it could also decay to $\mu^{+} \mu^{-}$. In this study, the $\mathrm{e}^{-}$beam energy is taken to be $100 \mathrm{MeV}$ and only the $e^{+} e^{-}$and $3 \gamma$ decay modes are considered. The expected DP decay length in the lab frame is [40]

$$
l_{\phi}=\frac{E_{k}}{m_{\phi}} \frac{1}{\Gamma_{\phi}}
$$




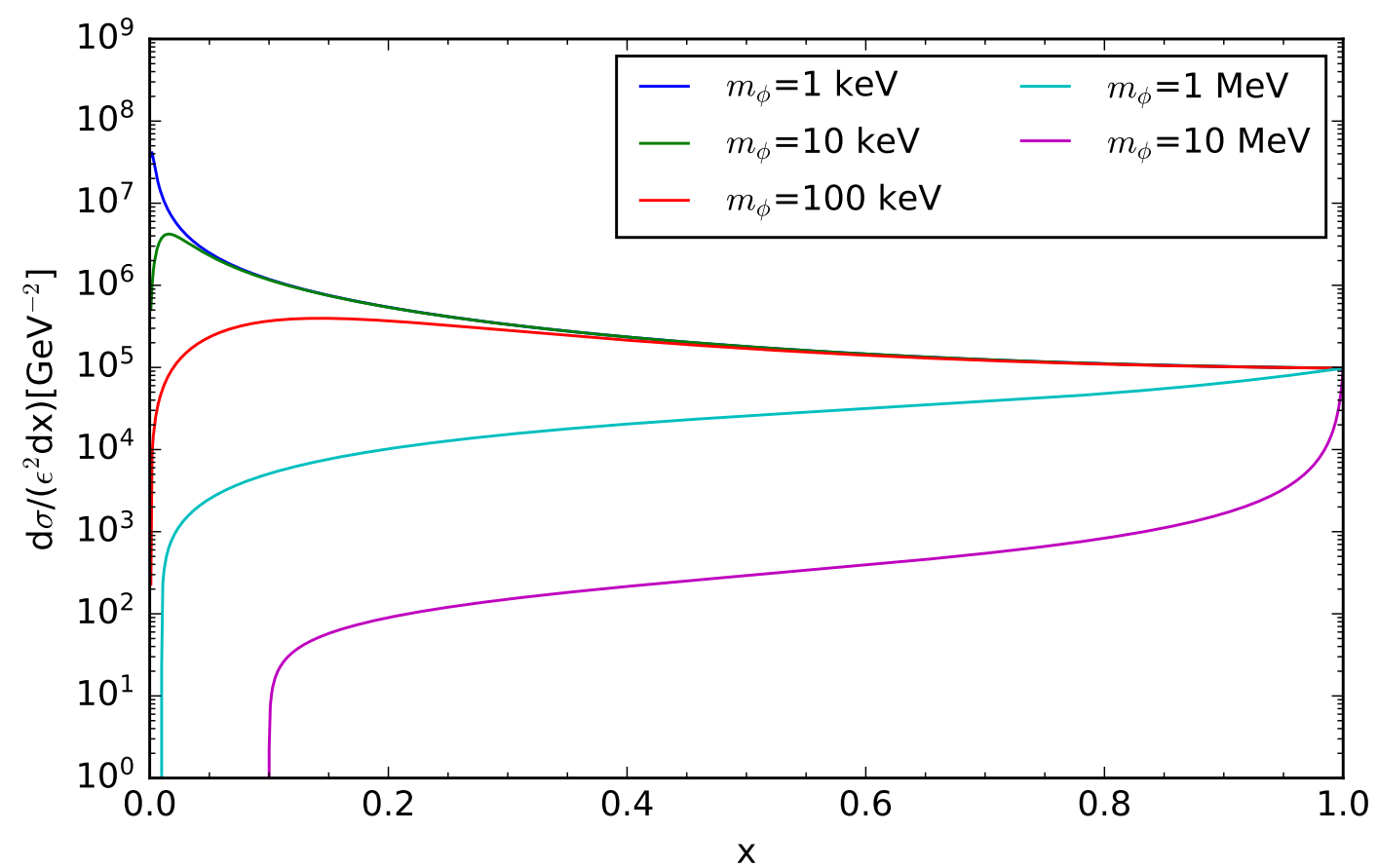

Figure 6. The generalized IWW differential cross-sections for DP production for a $100 \mathrm{MeV} e^{-}$ beam on a tungsten target for several different DP masses. Here $x$ is the fraction of DP energy relative to that of the $\mathrm{e}^{-}$beam energy. Note that here the differential cross-section is scaled by $1 / \epsilon^{2}$.

where $E_{k}$ is dark photon energy, and $\Gamma_{\phi}$ is the decay width of a dark photon and given in eqs. (3.4) and (3.5) for $e^{+} e^{-}$and $\gamma \gamma \gamma$ decays, respectively:

$$
\begin{aligned}
\Gamma\left(\phi \rightarrow e^{+} e^{-}\right) & =\epsilon^{2} \frac{\alpha}{2} m_{\phi}\left(1+\frac{2 m_{e}^{2}}{m_{\phi}^{2}}\right)\left(1-\frac{4 m_{e}^{2}}{m_{\phi}^{2}}\right)^{1 / 2}, \\
\Gamma(\phi \rightarrow \gamma \gamma \gamma) & =\epsilon^{2} \frac{\alpha^{4}}{2^{7} 3^{6} 5^{2} \pi^{3}} \frac{m_{\phi}^{9}}{m_{e}^{8}}\left[\frac{17}{5}+\frac{67}{42} \frac{m_{\phi}^{2}}{m_{e}^{2}}+\frac{128941}{246960} \frac{m_{\phi}^{4}}{m_{e}^{4}}+\mathcal{O}\left(\frac{m_{\phi}^{6}}{m_{e}^{6}}\right)\right] .
\end{aligned}
$$

Dark photons can also interact with electrons in the material of the target, shield, and detector, thereby producing real photons in a process similar to Compton scattering as shown in figure 7; this is called dark photon "absorption." The DP absorption length in the lab frame is given by

$$
\lambda=\frac{1}{n_{e} \sigma_{\mathrm{abs}}},
$$

where $n_{e}$ is the electron number density of the medium and $\sigma_{\text {abs }}$ is the total cross-section of the DP absorption and can be computed using eq. (36) in ref. [40].

\subsection{Expected number of dark photons}

Using the DP production cross-section for the darkstrahlung process in a thick target, and detection through visible decays and absorption interactions that are discussed above, the expected number of dark photons that are either absorbed or decay in the detector is 


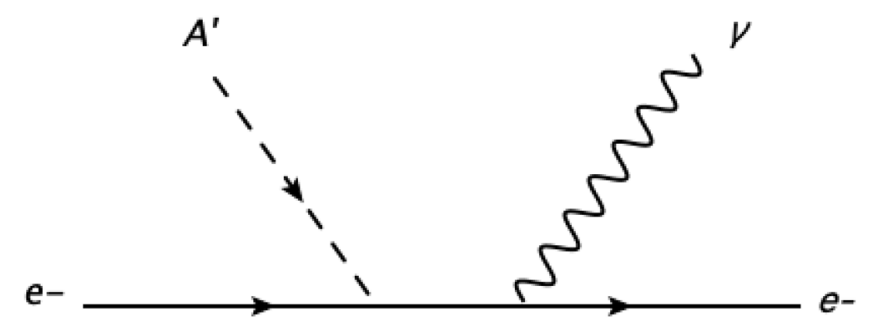

Figure 7. Dark photon absorption process similar to Compton scattering.

given by

$$
\begin{aligned}
N_{\phi} \approx & \frac{N_{e} X}{M} \int_{E_{\min }}^{E_{0}} d E \int_{x_{\min }}^{x_{\max }} d x \int_{0}^{T} d t I_{e}\left(E_{0}, E, t\right) \frac{d \sigma}{d x} \\
& \left.\times e^{-L_{\mathrm{sh}}\left(\frac{1}{l_{\phi}}+\frac{1}{\lambda_{\mathrm{sh}}}\right.}\right)\left(1-e^{-\left(L_{\mathrm{dec}} / l_{\phi}+L_{\mathrm{det}} / \lambda_{\mathrm{det}}\right)}\right),
\end{aligned}
$$

where $N_{e}$ is the total number of incoming electrons, $X$ the radiation length of the target material $\left(6.8 \mathrm{gm} / \mathrm{cm}^{2}\right.$ for tungsten); $M$ is the mass of target atom; $E_{0}$ is the incoming electron beam energy; $E_{\min }=m_{e}+\max \left(m_{\phi}, E_{\text {cut }}\right), x_{\min }=\frac{\max \left(m_{\phi}, E_{\text {cut }}\right)}{E}$, where $E_{\text {cut }}$ is the measured energy cutoff depending on the detector; $x_{\max }$ is very close to, but smaller than, 1 and is approximated to be $1-\frac{m_{e}}{E}$ if the DP and the initial and final electron states are collinear; $T=\rho L_{\mathrm{sh}} / X$ where $\rho$ is the density of the target; $l_{\phi}$ is the decay length of the DP in the lab frame; $\lambda_{\mathrm{sh}}\left(\lambda_{\mathrm{det}}\right)$ is the absorption length of the DP passing through target and shield (detector). Even though electrons enter the target with initial energy $E_{0}$, DP production could occur after some energy loss of the incoming electrons as they penetrate the target. This is taken into account with an analytic function $I_{e}\left(E_{0}, E, t\right)$ from ref. [40] that was derived in [41]:

$$
I_{e}\left(E_{0}, E, t\right)=\frac{\left(\ln \frac{E_{0}}{E}\right)^{\frac{4}{3} t-1}}{E_{0} \Gamma\left(\frac{4}{3} t\right)},
$$

where $t$ represents how many numbers of radiation length traversed by the electron before "darkstrahlung" occurs, $E$ is the $e^{-}$energy after $t$ radiation lengths and $\Gamma$ is the Gamma function. For the Yemilab neutrino detector, $E_{0}=100 \mathrm{MeV}, E_{\text {cut }}=200 \mathrm{keV}$, $N_{e}=1.97 \times 10^{23}$ for 1 year of operation with $100 \mathrm{~kW} e^{-}$beam power, $L_{\mathrm{sh}}=50 \mathrm{~cm}$, $L_{\mathrm{dec}}=L_{\mathrm{det}}=20 \mathrm{~m}$. (The decay distance, $L_{\mathrm{dec}}$, could be larger, depending on the distance between the shield and the detector, but the final sensitivity result does not change by much when $L_{\text {dec }}$ is doubled.)

Note also that DP signal loss can occur because of DP decay or absorption in the shield that is required to attenuate all of the standard model particle background; this loss in shield is accounted for by the $e^{-L_{\mathrm{sh}}\left(\frac{1}{l_{\phi}}+\frac{1}{\lambda_{\mathrm{sh}}}\right)}$ term in eq. (3.7). The DP detection probability via either decay or absorption is accounted for in the last term, the large brackets in eq. (3.7). 


\begin{tabular}{|c|c|c|c|c|}
\hline $\begin{array}{c}\text { Kinetic mixing } \\
\text { parameter } \epsilon\end{array}$ & $\begin{array}{c}\text { DP mass } \\
m_{\phi}(\mathrm{MeV})\end{array}$ & $\begin{array}{c}\text { Decay length } \\
l_{\phi}(\mathrm{m})\end{array}$ & $\begin{array}{c}\text { Absorption length } \\
\text { in shield: } \lambda_{\text {sh }}(\mathrm{m})\end{array}$ & $\begin{array}{c}\text { Absorption length } \\
\text { in target: } \lambda_{\text {det }}(\mathrm{m})\end{array}$ \\
\hline $10^{-3}$ & 0.1 & $\sim 10^{18}(3 \gamma)$ & $\sim 10^{5}$ & $\sim 10^{6}$ \\
$10^{-3}$ & 10 & $\sim 10^{-5}\left(e^{+} e^{-}\right)$ & $\sim 10^{5}$ & $\sim 10^{6}$ \\
\hline $10^{-5}$ & 0.1 & $\sim 10^{22}(3 \gamma)$ & $\sim 10^{9}$ & $\sim 10^{10}$ \\
$10^{-5}$ & 10 & $<1\left(e^{+} e^{-}\right)$ & $\sim 10^{9}$ & $\sim 10^{10}$ \\
\hline $10^{-8}$ & 0.1 & $\sim 10^{28}(3 \gamma)$ & $\sim 10^{15}$ & $\sim 10^{16}$ \\
$10^{-8}$ & 10 & $\sim 10^{5}\left(e^{+} e^{-}\right)$ & $\sim 10^{15}$ & $\sim 10^{16}$ \\
\hline
\end{tabular}

Table 1. Dark photon decay and absorption length scales for three different kinetic mixing parameters and for two different DP mass values. These length scales depend on the fractional of DP energy ( $\mathrm{x}$ ) and electron energy (E) at the DP point of production, while for the allowed ranges of $\mathrm{x}$ and $\mathrm{E}$ the length scale values given in the table do not change.

\section{Dark photon sensitivity at Yemilab}

Before obtaining the DP sensitivity at Yemilab, the DP decay and absorption lengths are compared to check which process is dominant for different DP masses and kinetic mixing parameters. Then rough estimation of background is discussed followed by DP sensitivities for decay-only, absorption-only, and both combined cases. For light DP $\left(m_{\phi}<2 m_{e}\right)$, oscillation between ordinary and dark photons is additionally discussed and the corresponding sensitivity is also obtained.

\subsection{Dark photon decay and absorption lengths}

Table 1 lists the DP decay and absorption lengths for $\epsilon=10^{-3}, 10^{-5}$ and $10^{-8}$ cases for $0.1 \mathrm{MeV}$ and $10 \mathrm{MeV}$ DP masses, which are taken as representative for the $m_{\phi}<2 m_{e}$ and $m_{\phi}>2 m_{e}$ cases corresponding to the $3 \gamma$ and $e^{+} e^{-}$decay modes, respectively. Note that the $3 \gamma$ decay lengths are very large while the $e^{+} e^{-}$decay lengths are more compatible with the detector size. However, for large values of $\epsilon$ (e.g., $\left.\epsilon=10^{-3}\right)$ the $e^{+} e^{-}$decay length becomes quite small $\left(\sim 10^{-5} \mathrm{~m}\right)$, and DP decays occur primarily in the shield, and well before they reach the detector. The absorption lengths in target/shield (both tungsten) and detector material (water, WbLS or LS) are similar but the length at the target/shield is an order of magnitude shorter due to its higher density. Note that the absorption length-scales are shorter than the $3 \gamma$ decay lengths and larger than those for the $e^{+} e^{-}$decays.

Figure 8 shows DP decay and absorption lengths for $\epsilon=10^{-3}, 10^{-5}$ and $10^{-8}$ cases for several different DP masses. The red horizontal line indicates detector diameter $(20 \mathrm{~m})$ where DP can either decay or be absorbed. If the DP decay and absorption lengths are longer or shorter than the detector size, the detection probability is suppressed.

\subsection{Background estimation}

Our strategy of DP search is to find excess of events by subtracting beam-OFF data from beam-ON data. In this way all background can be removed, but the background 

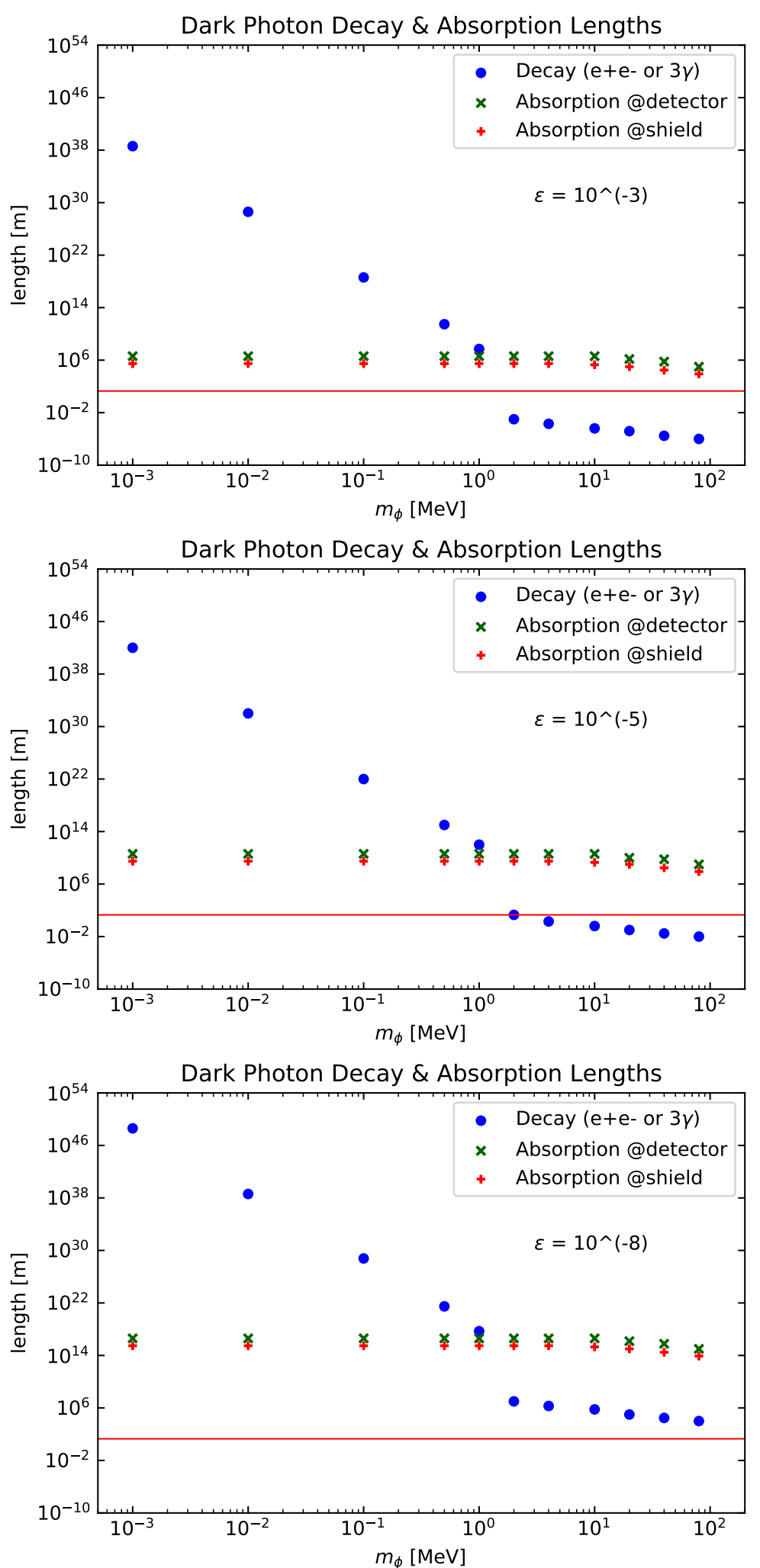

Figure 8. Dark photon decay and absorption lengths in the Yemilab setup for a $100 \mathrm{MeV}-100 \mathrm{~kW}$ $e^{-}$beam incident on a tungsten target $(50 \mathrm{~cm})$. The horizontal red line represents the diameter of the neutrino detector that is assumed in this study. 
subtraction introduces statistical uncertainty in the final number of signal events, affecting final sensitivity. A rough estimation of background is obtained to know its impact on DP sensitivity. Possible background sources are radiogenic, cosmic muon, neutron, solar $\nu$, atmospheric $\nu$, and beam related backgrounds.

All radiogenic background can be removed by requiring $5 \mathrm{MeV}$ energy threshold. Cosmic muon backgrounds can be suppressed by using a pulsed beam and adding an additional muon veto system that might be, for example, plastic scintillator modules on top of the detector plus an outer water Cherenkov veto detector that surrounds the inner detector [36]. Cosmogenic neutron backgrounds can be suppressed by tagging neutrons in a Gadoliniumloaded water, WbLS or LS detector. Neutrinos from ${ }^{8} \mathrm{~B}$ decays in the solar interior can contribute to the above $5 \mathrm{MeV}$ background but most of these could be removed by a directional veto and/or by requiring very forward vertex positions for DP signals in a water or WbLS detector. For an LS detector it would be challenging to apply directional veto. Rough estimation of the possible backgrounds assuming a LS detector is described below.

Cosmic muon, neutron, and ${ }^{8} \mathrm{~B}$ solar $\boldsymbol{\nu}$ background. Recently Borexino has measured ${ }^{8} \mathrm{~B}$ solar neutrino flux more precisely in ref. [42]. In table III of ref. [42], the ${ }^{8} \mathrm{~B}$ solar $\nu$ rate is listed as "bulk event" which also includes residual background originating from cosmic muon, and background from neutron capture-induced gamma is listed as "external component". These background rates are listed for low energy (HER-I) and high energy (HER-II) regions defined in ref. [42]. Thanks to the $5 \mathrm{MeV}$ energy threshold, the low energy background rate can be reduced to $\sim 1 / 3$ according to figure 2 of ref. [42], and ${ }^{208}$ Tl-related background at $2.6 \mathrm{MeV}$ is also removed. By combining HER-I and HER-II rates for the "bulk event" and "external component", we obtain $0.256 \mathrm{cpd} / 100$ ton, or equivalently 935 events per year for 1 kton fiducial volume ${ }^{1}$ for the Yemilab neutrino detector.

Atmospheric $\boldsymbol{\nu}$ background. Atmospheric neutrinos can contribute to background via a single $\pi^{0}$ production in $\nu+N \rightarrow \nu+N^{\prime}+\pi^{0}$ process. However, our electron beam energy $(100 \mathrm{MeV})$ is less than $\pi^{0}$ rest mass $(135 \mathrm{MeV})$, and therefore the two photons from $\pi^{0}$ decay would fire more PMTs than those from DP signal produced by our $e^{-}$beam. Thus, this background would be removed by requiring maximum number of fired PMTs or by requiring maximum visible energy. Total number of atmospheric background is estimated by Borexino measurement in ref. [43]. According table 2 in [43], total number of atmospheric background events in Borexino detector is estimated as 6.7 events/year/100 ton between $0.25 \mathrm{MeV}$ to $100 \mathrm{MeV}$ visible energy. This gives us 67 events/year/1 kton for the Yemilab neutrino detector as a conservative number because of the $5 \mathrm{MeV}$ energy threshold.

Beam-related background. There are two sources of beam-related background: one is neutrinos and the other is spallation neutrons. Firstly, neutrinos produced by NC or CC interactions $\left(e+N \rightarrow e+N+\bar{\nu}+\nu\right.$ or $\left.e+N \rightarrow \nu_{e}+N^{\prime}+X\right)$ in a beam target can mimic DP signal by neutrino scattering in our detector $\left(\nu+N \rightarrow \nu+N^{\prime}+X\right.$ or $\left.\nu+e \rightarrow \nu+e\right)$. The production cross-section of these neutrinos is found to be small, $\sim f b /$ nucleon [39] which corresponds to $\sim 10^{8}$ neutrinos per year for a tungsten target assuming $100 \mathrm{MeV}-100 \mathrm{~kW}$

\footnotetext{
${ }^{1}$ Fiducial volume should be optimized for physics analysis later.
} 
electron beam. The scattering probability for a neutrino to an electron in the detector is given by $\sigma_{\nu_{e}} \times n_{D} \times l_{D}$ where $\sigma_{\nu_{e}}$ is the cross-section of neutrino-electron scattering, and $n_{D}$ and $l_{D}$ are the number density for water and horizontal length $(20 \mathrm{~m})$ of the detector. The scattering cross-section sigma scales as $G_{F}^{2} \times E_{\nu} \times m_{e}$ where $G_{F}=1.66 \times 10^{-5} / \mathrm{GeV}^{2}$. Even though a maximum neutrino energy of $100 \mathrm{MeV}$ is considered, the scattering probability is very small, i.e. $\sim 10^{-18}$ scattering per neutrino resulting in $\sim 10^{-10}$ scatterings per year, so that this background is negligible. Secondly, spallation neutrons produced by inelastic scattering of electrons on nuclei target can mimic DP signal by the neutron scattering or neutrino (produced by beta decay of the neutrons) scattering in our detector. The inelastic cross-section of electrons on nuclei target can reach to $\sim m b$ level [39] resulting in a significant number of spallation neutrons and their beta-decay neutrinos. The spallation neutrons can be removed by placing few meters of rocks between beam-target and detector. The energy of the neutrinos produced from beta decay of neutrons is less than $5 \mathrm{MeV}$ and therefore they can be also removed by the $5 \mathrm{MeV}$ energy threshold we employed in our study.

\subsection{Dark photon sensitivity}

The expected number of dark photon signal events are obtained from eq. (3.7), where the energy threshold of the detector $\left(E_{\text {cut }}\right)$ is set at $5 \mathrm{MeV}$ in order to discriminate against all radiogenic backgrounds.

From the rough estimation of background discussed in the previous subsection, a total number of background events is expected to be $10^{3}$ events per year for 1 kton fiducial volume in the Yemilab neutrino detector. However, all of these background events can be removed by subtracting beam-OFF data from beam-ON data, $N_{\mathrm{DP}}=N_{\mathrm{ON}}-N_{\mathrm{OFF}}\left(\frac{T_{\mathrm{ON}}}{T_{\mathrm{OFF}}}\right) \approx 0$. From the subtraction of background events, statistical fluctuation for DP signal is given as:

$$
\Delta N_{\mathrm{DP}}=\sqrt{N_{\mathrm{ON}}+\left(\frac{T_{\mathrm{ON}}}{T_{\mathrm{OFF}}}\right)^{2} N_{\mathrm{OFF}}}=\sqrt{N_{\mathrm{ON}}\left(1+\frac{T_{\mathrm{ON}}}{T_{\mathrm{OFF}}}\right)}
$$

By taking long period of beam-OFF data, $\Delta N_{\mathrm{DP}} \approx \sqrt{N_{\mathrm{ON}}} \approx 32$. From this, one-sided $95 \%$ C.L. sensitivity is obtained as $1.645 \Delta N_{\mathrm{DP}} \approx 53$. Figure 9 shows excluded region sensitivities with $95 \%$ C.L. assuming either zero background (dark gray region under green) or $10^{3}$ background events per year (green region) in both beam-ON and OFF data with one year operation of $100 \mathrm{MeV}-100 \mathrm{~kW}$ electron beam. Depending on how well those estimated background to be further removed in the future, the $95 \%$ C.L. exclusion sensitivity shown in green region can be improved toward dark gray region. The upper plot in figure 9 shows the DP sensitivity for visible decays $\left(3 \gamma\right.$ or $e^{+} e^{-}$) only, where the sensitivity for $m_{\phi}<2 m_{e}$ decreases quickly for smaller $\epsilon$ values because the $3 \gamma$ decay length exceeds the size of the detector as shown in figure 8; there is no sensitivity for $\epsilon^{2}$ values between $10^{-6}$ and $10^{-10}$ for $m_{\phi}>2 m_{e}$ because of the short $e^{+} e^{-}$decay length, and below $\epsilon^{2} \sim 10^{-17}$ because of long $e^{+} e^{-}$decay length. The middle plot of figure 9 shows the DP sensitivity for the absorptiononly process. Not shown in figure 9 is that the number of $m_{\phi}>2 m_{e}$ DP events that are detectable via the absorption process, which is found to be much smaller than that of those detected via the decay process in the overlapping region of sensitivity in the parameter 
space. Note also that the sensitivity for the absorption process is nearly independent of DP mass for masses lighter than $2 m_{e}$. The lack of sensitivity for sub-MeV DPs for $\epsilon^{2}$ values below $1.5 \times 10^{-12}$ is because of their large absorption length. The bottom plot of figure 9 shows the DP sensitivity including both decay and absorption processes for a year-long run with a $100 \mathrm{MeV}-100 \mathrm{~kW} \mathrm{e}^{-}$beam. The $95 \%$-C.L. exclusion level for the $e^{+} e^{-}$decay mode for $m_{\phi}$ above $2 m_{e}$ is $\epsilon^{2}>4.8 \times 10^{-17}$ for zero background events, which is comparable to that for Super-Kamiokande's zero background assumption (see figure 4 in ref. [39]), and would have the world's best direct DP search sensitivity for $2 m_{e}<m_{\phi}<\sim 86 \mathrm{MeV}$ without considering SHiP. Once SHiP takes data up to $10^{20}$ POT (protons on target) [44], Yemilab sensitivity is best up to $\sim 50 \mathrm{MeV}(\sim 20 \mathrm{MeV})$ for zero $\left(10^{3}\right)$ background events. Figure 10 shows comparison of existing limits and some future projections of DP search for $m_{\phi}>2 m_{e}$. For sub-MeV DPs, the exclusion level is $\epsilon^{2}>1.5 \times 10^{-12}$ when considering only absorption process. Comparison of sub-MeV DP search is discussed in the following subsection with improved sensitivity by considering oscillation process.

\subsection{Oscillation between ordinary and dark photons}

Although it is not shown in figure 9 , the DP sensitivity for the absorption process extends down to very low DP masses, even to the sub-eV level. However, as discussed in refs. [27, 29], for $m_{\phi}<2 m_{e}$, oscillations between ordinary and dark photons (similar to neutrino oscillations) dominate. The oscillation probability is given in refs. [29, 49] to be

$$
\begin{aligned}
& P\left(\gamma \rightarrow A^{\prime}\right)=\epsilon^{2} \times \frac{m_{\phi}^{4}}{\left(\Delta m^{2}\right)^{2}+E_{\gamma}^{2} \Gamma^{2}}, \\
& P\left(A^{\prime} \rightarrow \gamma\right)=\epsilon^{2} \times \frac{m_{\phi}^{4}}{\left(\Delta m^{2}\right)^{2}+E_{\gamma}^{2} \Gamma^{2}} \times \Gamma L,
\end{aligned}
$$

where $\Delta m^{2}=\sqrt{\left(m_{\phi}^{2}-m_{\gamma}^{2}\right)^{2}+2 \epsilon^{2} m_{\phi}^{2}\left(m_{\phi}^{2}+m_{\gamma}^{2}\right)} \approx\left|m_{\phi}^{2}-m_{\gamma}^{2}\right|, m_{\gamma}=\sqrt{4 \pi \alpha n_{e} / m_{e}}$ is effective photon mass in matter, $E_{\gamma}$ and $\Gamma$ are ordinary photon energy and attenuation coefficient, respectively, and $L$ is the length of the detector in the beam direction.

In the case of the Yemilab neutrino detector, the oscillation would have to occur twice, one at production $\left(\gamma \rightarrow A^{\prime}\right)$ and the other at detection $\left(A^{\prime} \rightarrow \gamma\right)$. In this case, the oscillation probability is

$$
P\left(\gamma \leftrightarrow A^{\prime}\right)=\epsilon^{4} \times \frac{m_{\phi}^{8}}{\left(\left(m_{\phi}^{2}-m_{\gamma}^{\mathrm{W}}\right)^{2}+E_{\gamma}^{2} \Gamma_{\mathrm{W}}^{2}\right) \times\left(\left(m_{\phi}^{2}-m_{\gamma}^{\mathrm{H} 2 \mathrm{O} 2}\right)^{2}+E_{\gamma}^{2} \Gamma_{\mathrm{H} 2 \mathrm{O}}^{2}\right)} \times \Gamma_{\mathrm{H} 2 \mathrm{O}} L
$$

where, $m_{\gamma}^{\mathrm{W}}\left(m_{\gamma}^{\mathrm{H} 2 \mathrm{O}}\right)$ is an effective photon mass in tungsten (water), i.e. $80 \mathrm{eV}(21 \mathrm{eV})$, and $\Gamma_{\mathrm{W}}\left(\Gamma_{\mathrm{H} 2 \mathrm{O}}\right)$ is a photon attenuation coefficient in tungsten (water), where $\Gamma_{\mathrm{W}}^{-1} \simeq 1 \mathrm{~cm}$ $\left(\Gamma_{\mathrm{H} 2 \mathrm{O}}^{-1} \simeq 45 \mathrm{~cm}\right)$ at $E_{\gamma}=10 \mathrm{MeV}$ according to NIST database. In the following extreme 

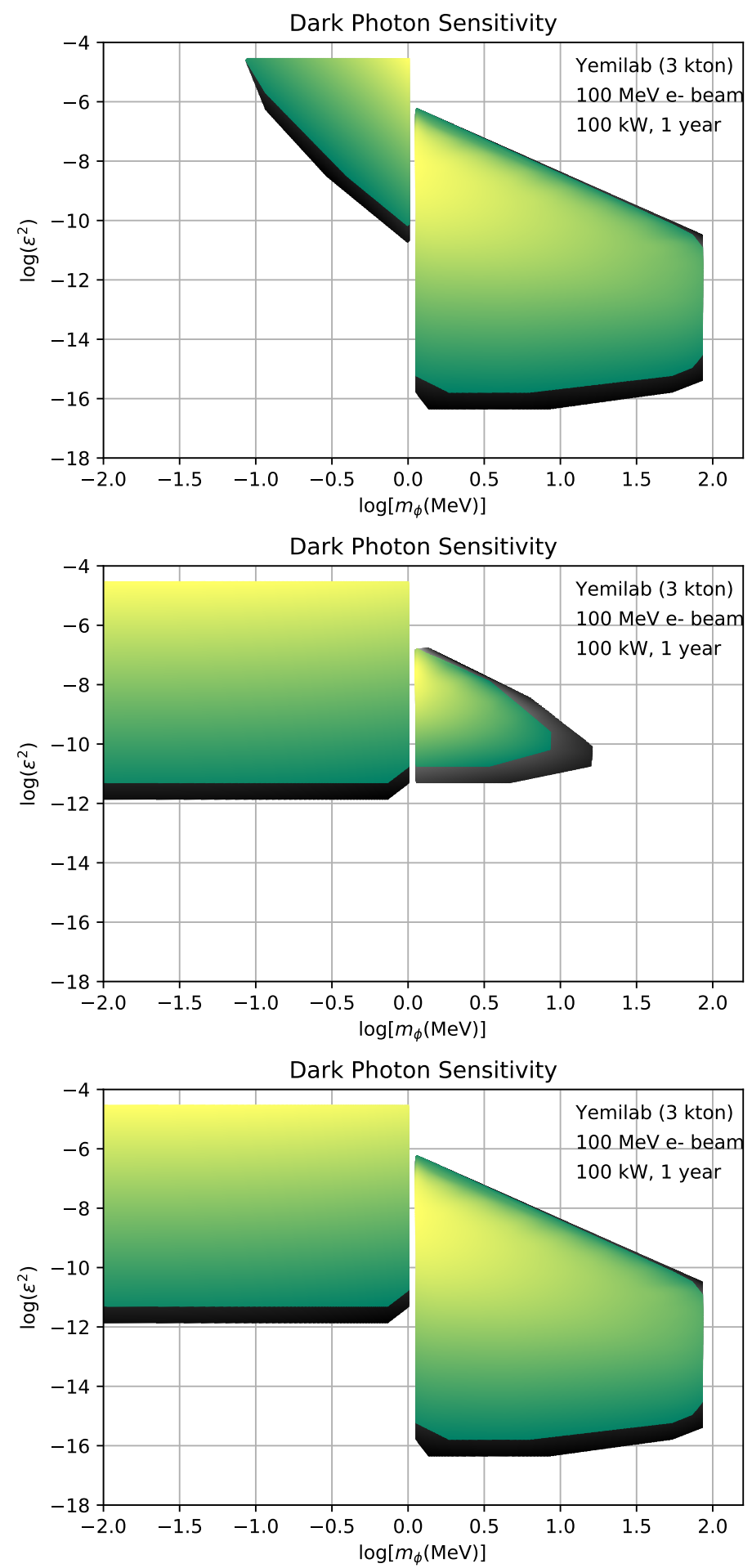

Figure 9. Dark photon excluded regions (95\% C.L. sensitivities) in the Yemilab neutrino detector for visible decays only (top), absorption-only (middle) and for both visible decays and absorption (bottom) for one year of data-taking with a $100 \mathrm{MeV}-100 \mathrm{~kW} e^{-}$beam on a tungsten target $(50 \mathrm{~cm})$. Dark gray (green) region is the sensitivity obtained for zero $\left(10^{3}\right)$ background events in both beam$\mathrm{ON}$ and $\mathrm{OFF}$ periods. 


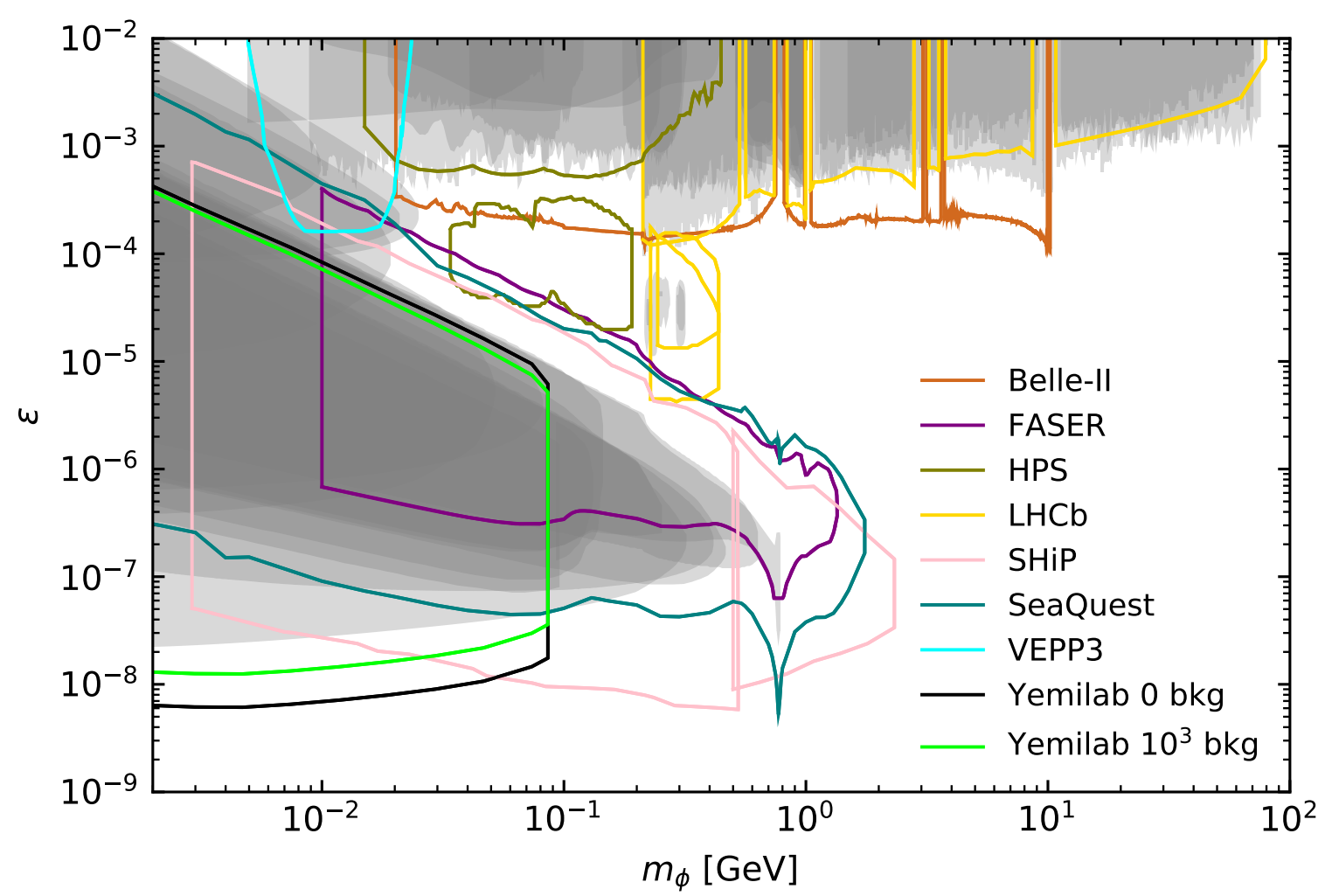

Figure 10. Comparison of existing limits (shaded gray) and some future projections (colored lines) for DP $\left(m_{\phi}>2 m_{e}\right)$ searches, drawn with darkcast framework [24] at https://gitlab.com/philten/ darkcast. Yemilab 95\% C.L. sensitivities with zero $\left(10^{3}\right)$ background events is shown in a black (green) line. Other future projections are drawn for Belle-II [45] (chocolate), FASER [25] (purple), HPS [46] (olive), LHCb [19] (gold), SHiP [44] (pink), SeaQuest [47] (teal), and VEPP3 [48] (cyan).

cases, the oscillation probability eq. (4.4) becomes

$$
\begin{array}{rlrl}
P\left(\gamma \leftrightarrow A^{\prime}\right) & =\epsilon^{4} \times \frac{m_{\phi}^{8}}{m_{\gamma}^{\mathrm{W} 4} \times m_{\gamma}^{\mathrm{H} 2 O}+} \times \Gamma_{\mathrm{H} 2 \mathrm{O}} L, & & \left(m_{\phi} \ll m_{\gamma}\right) \\
& =\epsilon^{4} \times \Gamma_{\mathrm{H} 2 \mathrm{O} L} L & & \left(m_{\phi} \gg m_{\gamma}\right) \\
& =\epsilon^{4} \times \frac{m_{\phi}^{8}}{E_{\gamma}^{2} \Gamma_{\mathrm{W}}^{2} \times\left(\left(m_{\gamma}^{\mathrm{W}} 2-m_{\gamma}^{\mathrm{H} 2 \mathrm{O} 2}\right)^{2}+E_{\gamma}^{2} \Gamma_{\mathrm{H} 2 \mathrm{O}}^{2}\right)} \times \Gamma_{\mathrm{H} 2 \mathrm{O} L,}, & \left(m_{\phi} \approx m_{\gamma}^{\mathrm{W}}\right) \\
& =\epsilon^{4} \times \frac{m_{\phi}^{8}}{\left(\left(m_{\gamma}^{\mathrm{H} 2 \mathrm{O} 2}-m_{\gamma}^{\mathrm{W} 2}\right)^{2}+E_{\gamma}^{2} \Gamma_{\mathrm{W}}^{2}\right) \times E_{\gamma}^{2} \Gamma_{\mathrm{H} 2 \mathrm{O}}^{2}} \times \Gamma_{\mathrm{H} 2 \mathrm{O} L} L, & & \left(m_{\phi} \approx m_{\gamma}^{\mathrm{H} 2 \mathrm{O}}\right)
\end{array}
$$

For $m_{\gamma}^{\mathrm{H} 2 \mathrm{O}}<m_{\phi}<m_{\gamma}^{\mathrm{W}}$, the oscillation probability is the same as eq. (4.4). At resonance ( $m_{\phi} \approx m_{\gamma}^{\mathrm{W}}$ or $m_{\gamma}^{\mathrm{H} 2 \mathrm{O}}$ ), the oscillation probability becomes maximum. Using eq. (4.4), the expected number of DP signal events from the oscillation is obtained as

$$
N_{\phi}^{\text {osc }} \approx N_{\mathrm{e}} \times \int_{E_{\gamma}^{\min }}^{E_{\max }^{\max }} d E_{\gamma} P\left(\gamma \leftrightarrow A^{\prime}\right) \int_{0}^{T} d t\left(I_{\gamma}^{(1)}\left(t, E_{\gamma}\right)+I_{\gamma}^{(2)}\left(t, E_{\gamma}\right)\right),
$$




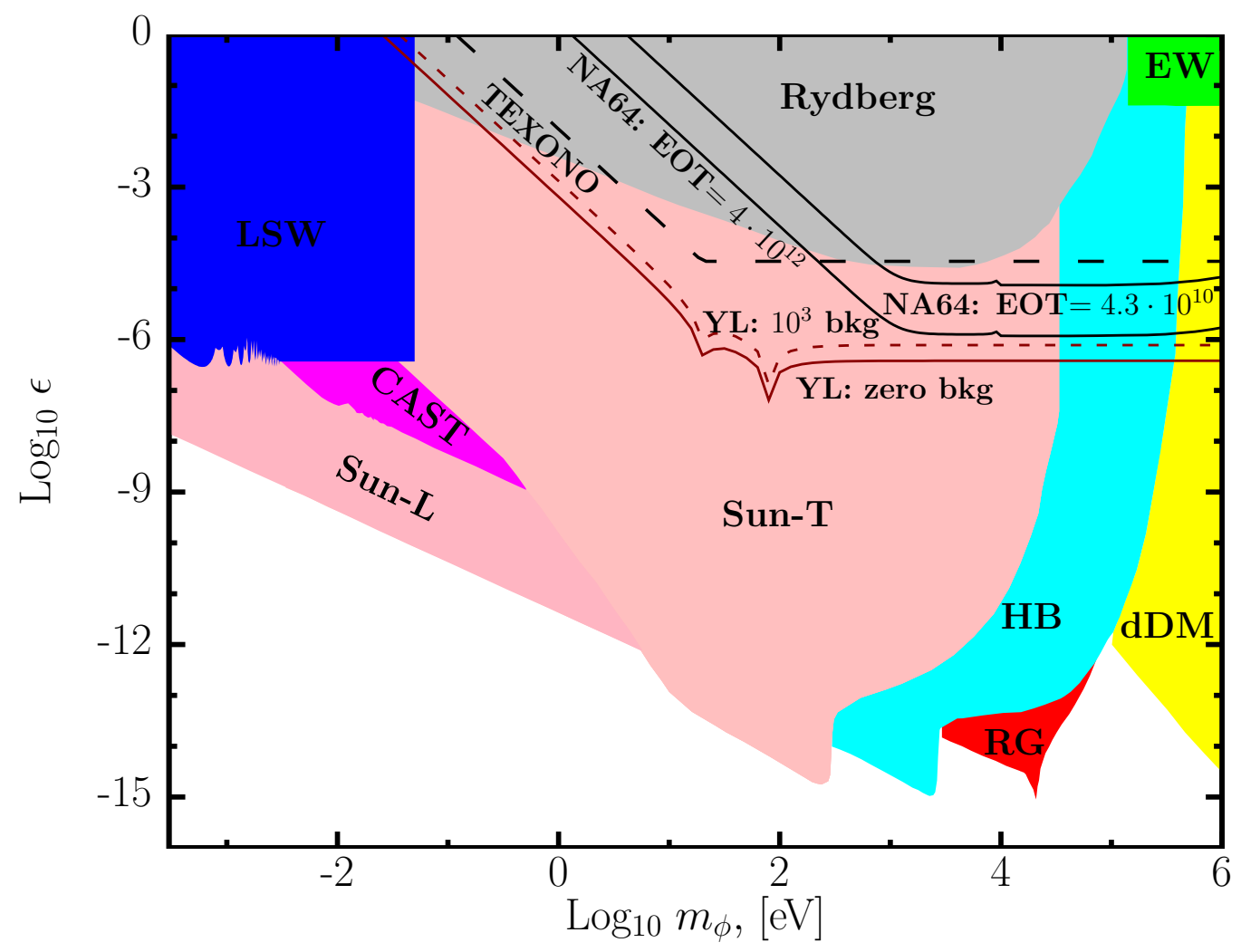

Figure 11. The dark photon sensitivity from the $\gamma \leftrightarrow A^{\prime}$ oscillation for $m_{\phi}<2 m_{e}$ at the Yemilab (YL) neutrino detector for one year of data taking with a $100 \mathrm{MeV}-100 \mathrm{~kW} e^{-}$beam (zero background: solid red line, $10^{3}$ background: dotted red line) on a tungsten target $(50 \mathrm{~cm})$, compared to those of the recent direct search experiments, TEXONO (dashed black line) [29] and NA64 (two solid black lines) [27]. Details on the limits from the helioscopic/astrophysical observations and the other experiments are found in [50-52].

where $I_{\gamma}^{(1)}$ and $I_{\gamma}^{(2)}$ are, respectively, the $1^{\text {st }}$ and $2^{\text {nd }}$ generations of photon flux in target per an incoming electron and given in the eqs. (24) and (29) of ref. [41]; $E_{\gamma}^{\min }=5 \mathrm{MeV}$ to remove radiogenic background and $E_{\gamma}^{\max } \approx E_{0}=100 \mathrm{MeV}$.

The $95 \%$ C.L. sub-MeV DP detection sensitivities for photon-DP oscillations determined from eq. (4.9) are shown in figure 11 for zero and $10^{3}$ background events with one year operation of $100 \mathrm{MeV}-100 \mathrm{~kW} e^{-}$beam. A comparison of figure 11 with figures 9 shows that the above $80 \mathrm{eV}$ mass DP sensitivity from oscillations is better than that for the absorption process; the best $95 \%$ C.L. direct DP search sensitivity, $\epsilon^{2}>1.5 \times 10^{-13}\left(6.1 \times 10^{-13}\right)$ for zero $\left(10^{3}\right)$ background events, is obtained in a year-long data-taking run with a $100 \mathrm{MeV}-$ $100 \mathrm{~kW} \mathrm{e}^{-}$beam on a thick tungsten target and the Yemilab neutrino detector.

\section{Summary}

Dark photon searches are the focus of a variety of experiments and have been invoked to explain a number of anomalies that have cropped up in (astro-)particle physics observations. Many of the best constraints, especially for sub-MeV dark photons, are from helioscopic 
or astrophysical observations. However, the helioscopic/astrophysical constraints depend, in a large part, on the choice of DP mean-free-path lengths inside stellar objects [53] and, therefore, direct search experiments at laboratories are absolutely necessary. Our study shows that a combination of a 3 kiloton-scale neutrino detector and an electron beam at Yemilab could constrain DP kinetic mixing parameters with the world's best direct search sensitivity for sub-MeV and above $\mathrm{MeV}$ DPs produced via darkstrahlung $\left(e^{-}+Z \rightarrow e^{-}+Z+A^{\prime}\right)$ or oscillations $\left(\gamma \rightarrow A^{\prime}\right)$. In our study, DP is searched by observing excess of events in beam-ON data subtracted by beam-OFF data. By detecting DPs via their absorption $\left(A^{\prime}+e^{-} \rightarrow \gamma+e^{-}\right)$or oscillations $\left(A^{\prime} \rightarrow \gamma\right)$ processes, a 95\% C.L. direct search sensitivity for $80 \mathrm{eV}<m_{\phi}<2 m_{e} \mathrm{MeV}$ DP of $\epsilon^{2}>1.5 \times 10^{-13}\left(6.1 \times 10^{-13}\right)$ could be achieved with one year of operation of a $100 \mathrm{MeV}-100 \mathrm{~kW}$ electron beam on a thick tungsten target for zero $\left(10^{3}\right)$ background events in both beam-ON and OFF periods. The best sensitivities for sub-MeV DPs are achieved by exploiting the oscillation between ordinary and dark photons. At the peaks of the oscillation resonances, i.e. $m_{\phi}=21 \mathrm{eV}$ (in water) and $80 \mathrm{eV}$ (in tungsten), the sensitivities are enhanced as shown in figure 11, and $\epsilon^{2} \sim \mathcal{O}\left(10^{-15}\right)$ at $m_{\phi}=80 \mathrm{eV}$ for zero background events. The dark photon sensitivity for masses below the $m_{\phi}<21 \mathrm{eV}$ resonance peak rapidly decreases as $\epsilon^{4} \propto m_{\phi}^{-8}$ due to the oscillation. Sub$\mathrm{MeV}$ dark photon detection via decays to $3 \gamma$ are highly suppressed because of the very long decay lengths. For $2 m_{e}<m_{\phi}<\sim 86 \mathrm{MeV}$ DPs, the direct search sensitivity for the kinetic mixing parameters using visible decays $\left(A^{\prime} \rightarrow e^{+} e^{-}\right)$is $\epsilon^{2}>\mathcal{O}\left(10^{-17}\right)\left(\mathcal{O}\left(10^{-16}\right)\right)$ at the $95 \%$ C.L. for zero $\left(10^{3}\right)$ background events using the $100 \mathrm{MeV}-100 \mathrm{~kW} e^{-}$beam; a sensitivity that is comparable to that of Super-K for the same zero background assumption as Super-K. With a higher energy electron beam, the sensitivity beyond $86 \mathrm{MeV}$ DPs could also be explored but, it may not be practical to accommodate such a facility in the current Yemilab design configuration.

\section{Acknowledgments}

The authors are grateful to D. Gorbunov and S. Demidov for very useful discussions about the possibilities of oscillations between ordinary and dark photons as well as providing us with the data points incorporated in figure 11. We also thank H.S. Lee and H.K. Park for visiting IBS to share their expertise on dark photons and thank T. Terada for help with the Mathematica calculations. Our special thanks goes to S. Olsen for reading our manuscript carefully and for giving us valuable feedback. This work was supported by the National Research Foundation of Korea (NRF) grant funded by the Korea Ministry of Science and ICT (MSIT) (No. 2017R1A2B4012757, IBS-R016-D1-2019-b01) and IBS-R016-D1.

Open Access. This article is distributed under the terms of the Creative Commons Attribution License (CC-BY 4.0), which permits any use, distribution and reproduction in any medium, provided the original author(s) and source are credited. 


\section{References}

[1] A.J. Krasznahorkay et al., Observation of Anomalous Internal Pair Creation in Be8: A Possible Indication of a Light, Neutral Boson, Phys. Rev. Lett. 116 (2016) 042501 [arXiv: 1504.01527] [INSPIRE].

[2] A.J. Krasznahorkay et al., New evidence supporting the existence of the hypothetic X17 particle, arXiv:1910.10459 [INSPIRE].

[3] J.D. Bjorken, R. Essig, P. Schuster and N. Toro, New Fixed-Target Experiments to Search for Dark Gauge Forces, Phys. Rev. D 80 (2009) 075018 [arXiv: 0906.0580] [InSPIRE].

[4] J.D. Bjorken et al., Search for Neutral Metastable Penetrating Particles Produced in the SLAC Beam Dump, Phys. Rev. D 38 (1988) 3375 [inSPIRE].

[5] A. Bross, M. Crisler, S.H. Pordes, J. Volk, S. Errede and J. Wrbanek, A Search for Shortlived Particles Produced in an Electron Beam Dump, Phys. Rev. Lett. 67 (1991) 2942 [inSPIRE].

[6] A1 collaboration, Search for Light Gauge Bosons of the Dark Sector at the Mainz Microtron, Phys. Rev. Lett. 106 (2011) 251802 [arXiv:1101.4091] [INSPIRE].

[7] APEX collaboration, Search for a New Gauge Boson in Electron-Nucleus Fixed-Target Scattering by the APEX Experiment, Phys. Rev. Lett. 107 (2011) 191804 [arXiv:1108.2750] [INSPIRE].

[8] R. Essig, P. Schuster, N. Toro and B. Wojtsekhowski, An Electron Fixed Target Experiment to Search for a New Vector Boson A' Decaying to $e^{+} e^{-}$, JHEP 02 (2011) 009 [arXiv: 1001.2557] [INSPIRE].

[9] KLOE-2 collaboration, Limit on the production of a light vector gauge boson in $\phi$ meson decays with the KLOE detector, Phys. Lett. B $\mathbf{7 2 0}$ (2013) 111 [arXiv:1210.3927] [INSPIRE].

[10] E. Izaguirre, G. Krnjaic, P. Schuster and N. Toro, New Electron Beam-Dump Experiments to Search for MeV to few-GeV Dark Matter, Phys. Rev. D 88 (2013) 114015 [arXiv:1307.6554] [INSPIRE].

[11] KLOE-2 collaboration, Search for light vector boson production in $e^{+} e^{-} \rightarrow \mu^{+} \mu^{-} \gamma$ interactions with the KLOE experiment, Phys. Lett. B 736 (2014) 459 [arXiv:1404.7772] [INSPIRE].

[12] B. Batell, R. Essig and Z. Surujon, Strong Constraints on Sub-GeV Dark Sectors from SLAC Beam Dump E137, Phys. Rev. Lett. 113 (2014) 171802 [arXiv:1406.2698] [InSPIRE].

[13] H. Merkel et al., Search at the Mainz Microtron for Light Massive Gauge Bosons Relevant for the Muon g-2 Anomaly, Phys. Rev. Lett. 112 (2014) 221802 [arXiv:1404.5502] [InSPIRE].

[14] J. Blümlein and J. Brunner, New Exclusion Limits on Dark Gauge Forces from Proton Bremsstrahlung in Beam-Dump Data, Phys. Lett. B 731 (2014) 320 [arXiv:1311.3870] [INSPIRE].

[15] NA48/2 collaboration, Search for the dark photon in $\pi^{0}$ decays, Phys. Lett. $B \mathbf{7 4 6}$ (2015) 178 [arXiv: 1504.00607] [INSPIRE].

[16] A. Anastasi et al., Limit on the production of a low-mass vector boson in $\mathrm{e}^{+} \mathrm{e}^{-} \rightarrow \mathrm{U} \gamma$, $\mathrm{U} \rightarrow \mathrm{e}^{+} \mathrm{e}^{-}$with the KLOE experiment, Phys. Lett. B 750 (2015) 633 [arXiv:1509.00740] [INSPIRE].

[17] P. Ilten, J. Thaler, M. Williams and W. Xue, Dark photons from charm mesons at LHCb, Phys. Rev. D 92 (2015) 115017 [arXiv:1509.06765] [InSPIRE]. 
[18] KLOE-2 collaboration, Limit on the production of a new vector boson in $\mathrm{e}^{+} \mathrm{e}^{-} \rightarrow \mathrm{U} \gamma$, $U \rightarrow \pi^{+} \pi^{-}$with the KLOE experiment, Phys. Lett. B 757 (2016) 356 [arXiv:1603.06086] [INSPIRE].

[19] P. Ilten, Y. Soreq, J. Thaler, M. Williams and W. Xue, Proposed Inclusive Dark Photon Search at LHCb, Phys. Rev. Lett. 116 (2016) 251803 [arXiv: 1603.08926] [InSPIRE].

[20] BDX collaboration, Dark matter search in a Beam-Dump eXperiment (BDX) at Jefferson Lab: an update on PR12-16-001, arXiv:1712.01518 [INSPIRE].

[21] BABAR collaboration, Search for Invisible Decays of a Dark Photon Produced in $e^{+} e^{-}$ Collisions at BaBar, Phys. Rev. Lett. 119 (2017) 131804 [arXiv:1702.03327] [InSPIRE].

[22] DarkLight collaboration, Searching for a dark photon with DarkLight, Nucl. Instrum. Meth. A 865 (2017) 125 [INSPIRE].

[23] LHCb collaboration, Search for Dark Photons Produced in $13 \mathrm{TeV}$ pp Collisions, Phys. Rev. Lett. 120 (2018) 061801 [arXiv:1710.02867] [INSPIRE].

[24] P. Ilten, Y. Soreq, M. Williams and W. Xue, Serendipity in dark photon searches, JHEP 06 (2018) 004 [arXiv : 1801.04847] [inSPIRE].

[25] FASER collaboration, FASER's physics reach for long-lived particles, Phys. Rev. D 99 (2019) 095011 [arXiv: 1811.12522] [INSPIRE].

[26] NA64 collaboration, Improved limits on a hypothetical X(16.7) boson and a dark photon decaying into $e^{+} e^{-}$pairs, Phys. Rev. D 101 (2020) 071101 [arXiv:1912.11389] [InSPIRE].

[27] S. Demidov, S. Gninenko and D. Gorbunov, Light hidden photon production in high energy collisions, JHEP 07 (2019) 162 [arXiv: 1812.02719] [INSPIRE].

[28] H. Park, Detecting Dark Photons with Reactor Neutrino Experiments, Phys. Rev. Lett. 119 (2017) 081801 [arXiv:1705.02470] [INSPIRE].

[29] M. Danilov, S. Demidov and D. Gorbunov, Constraints on hidden photons produced in nuclear reactors, Phys. Rev. Lett. 122 (2019) 041801 [arXiv: 1804.10777] [INSPIRE].

[30] J. Beacham et al., Physics Beyond Colliders at CERN: Beyond the Standard Model Working Group Report, J. Phys. G 47 (2020) 010501 [arXiv: 1901.09966] [INSPIRE].

[31] A. Filippi and M. De Napoli, Searching in the dark: the hunt for the dark photon, Rev. Phys. 5 (2020) 100042 [arXiv:2006.04640] [INSPIRE].

[32] R. Essig et al., Working Group Report: New Light Weakly Coupled Particles, in Community Summer Study 2013: Snowmass on the Mississippi, (2013) [arXiv:1311.0029] [INSPIRE].

[33] LHCb collaboration, Search for $A^{\prime} \rightarrow \mu^{+} \mu^{-}$Decays, Phys. Rev. Lett. 124 (2020) 041801 [arXiv: 1910.06926] [INSPIRE].

[34] COSINE-100 collaboration, Search for a Dark Matter-Induced Annual Modulation Signal in NaI(Tl) with the COSINE-100 Experiment, Phys. Rev. Lett. 123 (2019) 031302 [arXiv: 1903.10098] [INSPIRE].

[35] G.B. Kim et al., Novel measurement method of heat and light detection for neutrinoless double beta decay, Astropart. Phys. 91 (2017) 105 [InSPIRE].

[36] S.-H. Seo, Neutrino Telescope at Yemilab, Korea, arXiv:1903.05368 [InSPIRE].

[37] RENO collaboration, Spectral Measurement of the Electron Antineutrino Oscillation Amplitude and Frequency using 500 Live Days of RENO Data, Phys. Rev. D 98 (2018) 012002 [arXiv: 1610.04326] [INSPIRE]. 
[38] NEOS collaboration, Sterile Neutrino Search at the NEOS Experiment, Phys. Rev. Lett. 118 (2017) 121802 [arXiv: 1610.05134] [INSPIRE].

[39] E. Izaguirre, G. Krnjaic and M. Pospelov, MeV-Scale Dark Matter Deep Underground, Phys. Rev. D 92 (2015) 095014 [arXiv: 1507.02681] [InSPIRE].

[40] Y.-S. Liu and G.A. Miller, Validity of the Weizsäcker-Williams approximation and the analysis of beam dump experiments: Production of an axion, a dark photon, or a new axial-vector boson, Phys. Rev. D 96 (2017) 016004 [arXiv:1705.01633] [INSPIRE].

[41] Y.-S. Tsai and V. Whitis, Thick target bremsstrahlung and target consideration for secondary particle production by electrons, Phys. Rev. 149 (1966) 1248 [INSPIRE].

[42] BoreXino collaboration, Improved measurement of ${ }^{8} B$ solar neutrinos with $1.5 \mathrm{kt} \cdot y$ of Borexino exposure, Phys. Rev. D 101 (2020) 062001 [arXiv:1709.00756] [InSPIRE].

[43] V.S. Atroshchenko and E.A. Litvinovich, Estimation of atmospheric neutrinos background in Borexino, J. Phys. Conf. Ser. 675 (2016) 012014 [INSPIRE].

[44] S. Alekhin et al., A facility to Search for Hidden Particles at the CERN SPS: the SHiP physics case, Rept. Prog. Phys. 79 (2016) 124201 [arXiv: 1504.04855] [InSPIRE].

[45] Belle-II collaboration, The Belle II Physics Book, PTEP 2019 (2019) 123C01 [Erratum ibid. 2020 (2020) 029201] [arXiv: 1808.10567] [INSPIRE].

[46] HPS collaboration, The Heavy Photon Search beamline and its performance, Nucl. Instrum. Meth. A 859 (2017) 69 [arXiv:1612.07821] [INSPIRE].

[47] S. Gardner, R.J. Holt and A.S. Tadepalli, New Prospects in Fixed Target Searches for Dark Forces with the SeaQuest Experiment at Fermilab, Phys. Rev. D 93 (2016) 115015 [arXiv: 1509.00050] [INSPIRE].

[48] B. Wojtsekhowski, D. Nikolenko and I. Rachek, Searching for a new force at VEPP-3, arXiv: 1207.5089 [INSPIRE].

[49] J. Redondo, ATLAS of solar hidden photon emission, JCAP 07 (2015) 024 [arXiv: 1501.07292] [INSPIRE].

[50] J. Redondo and G. Raffelt, Solar constraints on hidden photons re-visited, JCAP 08 (2013) 034 [arXiv: 1305.2920] [INSPIRE].

[51] J.L. Hewett et al., Fundamental Physics at the Intensity Frontier, in Workshop on Fundamental Physics at the Intensity Frontier, (2012) [DOI] [arXiv:1205.2671] [INSPIRE].

[52] H. An, M. Pospelov, J. Pradler and A. Ritz, Direct Detection Constraints on Dark Photon Dark Matter, Phys. Lett. B $\mathbf{7 4 7}$ (2015) 331 [arXiv:1412.8378] [InSPIRE].

[53] J. Redondo, Helioscope Bounds on Hidden Sector Photons, JCAP 07 (2008) 008 [arXiv:0801.1527] [INSPIRE]. 